\title{
Blockchain Based Secure Traffic Police Assistant System
}

\author{
Dipti Pawade*, Avani Sakhapara \\ Assistant Professor, Department of Information Technoloogy, K J Somaiya College of Engineering, Mumbai, India \\ E-mail: diptipawade@somaiya.edu*
}

Raj shah, Siby Thampi, Vignesh Vaid

Student, Department of Information Technoloogy, K J Somaiya College of Engineering, Mumbai, India

Received:30 June 2020; Accepted: 15 July 2020; Published: 08 December 2020

\begin{abstract}
There are large numbers of vehicles in the populated country like India. It's a very common scenario that traffic police came across some vehicle random vehicle and had some doubt in mind but do not have in hand information about that vehicle and end up leaving that thought. Sometimes this may result in some disaster. With the advent of technology, there are mobile applications and web based systems are available to ease up the process by which traffic police can fine the vehicle owner or people can pay the fine online. But yet there is no system is available through which traffic police can get all the details about the particular vehicle. This motivated us to design and developed an application thorough which traffic police can get all the information right from owner of the vehicle to its RC book and insurance status on just one click. Looking at the chances of data tampering, we have also played an attention to the data security and used blockchain for creating distributed, robust and tempered proof system. In this paper we have discussed traffic police assistance system, which can scan the vehicle number plate, identify the number and provide the all the information and documents stored against that vehicle number. To address the issue of data security and alteration of sensitive data blockchain is used so that any alteration can be monitored. As the complete information process is dependent on how correctly the vehicle number is identified, so the number plate recognition module is tested thoroughly under various conditions. Finally user feedback is taken and analyzed to evaluate the feasibility and usability of the proposed application.
\end{abstract}

Index Terms: Image to text conversion, Number plate recognition, RTO data, Blockch ain, IPFS network.

\section{Introduction}

In a large country like India it is very difficult to track vehicle information. It's a usual scenario for vehicles to be parked in the no parking zone or vehicles violating traffic rules. Even there are certain suspicious cases; where vehicles are parked for day long and no one claims it. In all such cases, it is very difficult for traffic police to get vehicle information as they have to follow a very tedious method. The conventional methodology to acquire data about any vehicle is as follows:

1) Noting down the license plate number of the vehicle

2) Reporting to RTO regarding the suspicious activity or law violating activity of the vehicle

3) Receiving necessary information from RTO

There are some cases where criminals have used vehicle with fake license number plate and police authority came to know about this fact after few days as they had to follow the manual procedure. Now currently at some places the traffic police have started using the e-challan system [1]. Here a CCTV camera continuously records footage of the ongoing traffic. If a motorist breaks any traffic rules, this act gets recorded in the footage. The police then try to extract the license plate number from the vehicle's screen-shot captured from the CCTV footage and the offense is registered in the records. The traffic police then co-ordinates with the Regional Transport Office (RTO) to find the details of the vehicle and its owner like the registration number of the vehicle, the name and address of the vehicle owner, make and model of the vehicle, etc. As soon as the traffic police receive these details, an SMS is sent on the registered phone number of the violator. This SMS contains the time, date and location of the offense. The constraint of this system is that the time required in processing a particular CCTV footage and fetch the information out of it is still on higher side. For the ease of citizens, there is RTO related application called MumTrafficApp[2] which is developed. This citizen centric application basically helps citizens to keep a track of the traffic challans issued for their vehicles. Other features available in this application are provisions for the violator to view the evidences based on which challan is generated, 
online challan payment and traffic regulation rule book. Also citizens can register complaints if somebody violates the traffic rules. Similarly for the ease of traffic police, technology assisted solutions are being explored and experimented. In one of such experiments, on a pilot basis, in various states of India, traffic police have started using body worn cameras to record conversation between traffic violators and cops[3,4,5]. This kind of arrangement is helpful to have a proof against what kind of conversation happed between them. These videos are sent to control room where they are stored and analyzed further. This system is useful in collecting the evidences for allegations for misbehavior of citizens with traffic police or vice-a-versa. But for a scenario where traffic police just wants to know the vehicle information, it is not handy.

So to make this process more convenient and quick, we propose an application called Car-Docs wherein traffic police can scan the vehicle license plate number using smart phone and instantly all the details including the owner name and associated documents would be available to him at that point itself. The research objectives are as follows:

- Develop an efficient system to identify the vehicle number

- Provide easy access of all information related to particular vehicle

- Maintain data security

\section{Literature Survey}

In this section, we have studied the various attempts made by researchers to design and develop a technology assisted solution for the ease of traffic police and RTO. To start with, Avanish et. al. [6] proposed a system where traffic police officer scans the license of the driver with the help of a Smart Card Reader. Here the license is kind of a smart card which contains an embedded micro module consisting of a microprocessor and memory. As the officer scans the license, the application installed on the officer's device displays the details of the driver. Meanwhile, the system verifies the license and on the basis of violated rules selected by the officer penalty of corresponding amount is charged to the driver and the record of the same is updated on the main server. Limitations of this system are

- Additional cost incurred due to overhead of designing smart card based licenses.

- In densely populated country like India, it is not feasible to replace all regular licenses with new one.

Smart RTO web and android application, discussed in [7], includes user module, traffic module and RTO admin module. In this system, user module consists of forms i.e. license registration, vehicle registration and insurance forms which are to be filled by user. The traffic module focuses mainly on providing the information to the traffic police officers which consists of license check, vehicle information check and insurance check and also generate fines in case some driver violates the rules. And the RTO admin verifies the information filled within the forms by the user to generate license and vehicle number. Limitation of the system is data security measures to avoid data leakage or alteration is not discussed

E-RTO management system [8] includes registration of vehicle and storing of related data such as insurance, license plate number, etc. of the vehicle and its owner. It also includes authentication of vehicle using RFIDs which are issued by the RTO after successful verification. Here, RTO is a power user who has the power to verify and process the data provided by the user (vehicle owner/driver). Manjunath et. al. [9] proposed a system which provides services using cellular phones. The architecture consists of three modules namely RTO module, Flex module and Court module. In this architecture, RTO module is used to store data such as user license, vehicle information, vehicle insurance information, and the vehicle emission information. Flex module is used by the Traffic Police officer to perform verification of Vehicle and Driver License information. It also provides a facility to generate fine amount on the same system. Court module contains information regarding Officer Id, Date, Vehicle Registration, list of Offences, Fine Amount, and Payment Status. A. Choudhury et. al. [10] discussed the number plate identification method based on horizontal and vertical edge processing and zone detection. This method is proved to be an effective way to predict the number of the vehicle from the image of number plate capture from any angle or with any background. Ayman Rabee et. al. [11] has put forth a robust license plate recognition system. In pre-processing part, the color image is converted into gray scale using weighted sum method. Then contrast enhancement is applied for extracting License plate number from poor illumination which is then followed by mathematical morphology and segmentation using eight connected component algorithm. Then character segmentation is carried out and finally characters are recognized using support vector machines (SVMs). Here author has achieved the character recognition accuracy of $97.89 \%$. RTO verification system is proposed by Amruta et. al. [12] in which the authors have suggested to maintain centralized RTO database containing the information about vehicle and drivers. Using an android application, traffic police can retrieve vehicle and license information. They have not considered the data security aspect of centralized database. The proposed system [13] first detects for any vehicle which violates traffic rules and then captures the vehicle image. From the captured image, using image segmentation technique the license number plate region will be extracted. And the technique used for the character recognition on number plate is Optical character recognition. The system is implemented and simulated using Matlab. Using feedback system, the extracted license plate number is then given to GSM modem for further SMS feedback system to the user and concerned authority. 
From the literature survey it has been observed that many researchers has attempted to provide solution for RTO data management but still there is a need of system through which vehicle information can be accessed at real time. Also there is a need to address measures to be carried out to maintain information security and integrity. We have proposed a system to address all these requirements.

\section{Implementation overview}

Car-Docs portal provides an interface to upload car documents so that the process of accessing them for traffic policemen or any other authorized personnel becomes hassle-free, secure and paperless. Such type of system is very much required so that traffic police can get information about any suspicious vehicle on one click.

Our application consists of two interfaces:

A. Information upload interface: is used by the authorized user so that in future they can upload the important documents like vehicle insurance papers directly on portal.

B. Information access interface: is designed for the traffic police so that they can directly scan the vehicle number plate and get information about that vehicle.

\subsection{Information upload interface}

Figure 1. shows the overall flow of module to upload information. To upload any information, initially the user needs to go through the signup process where the user is prompted to enter basic information like name, address, phone number etc. Once the user is registered successfully, thereafter the user can log in into the system with registered emailid and password.

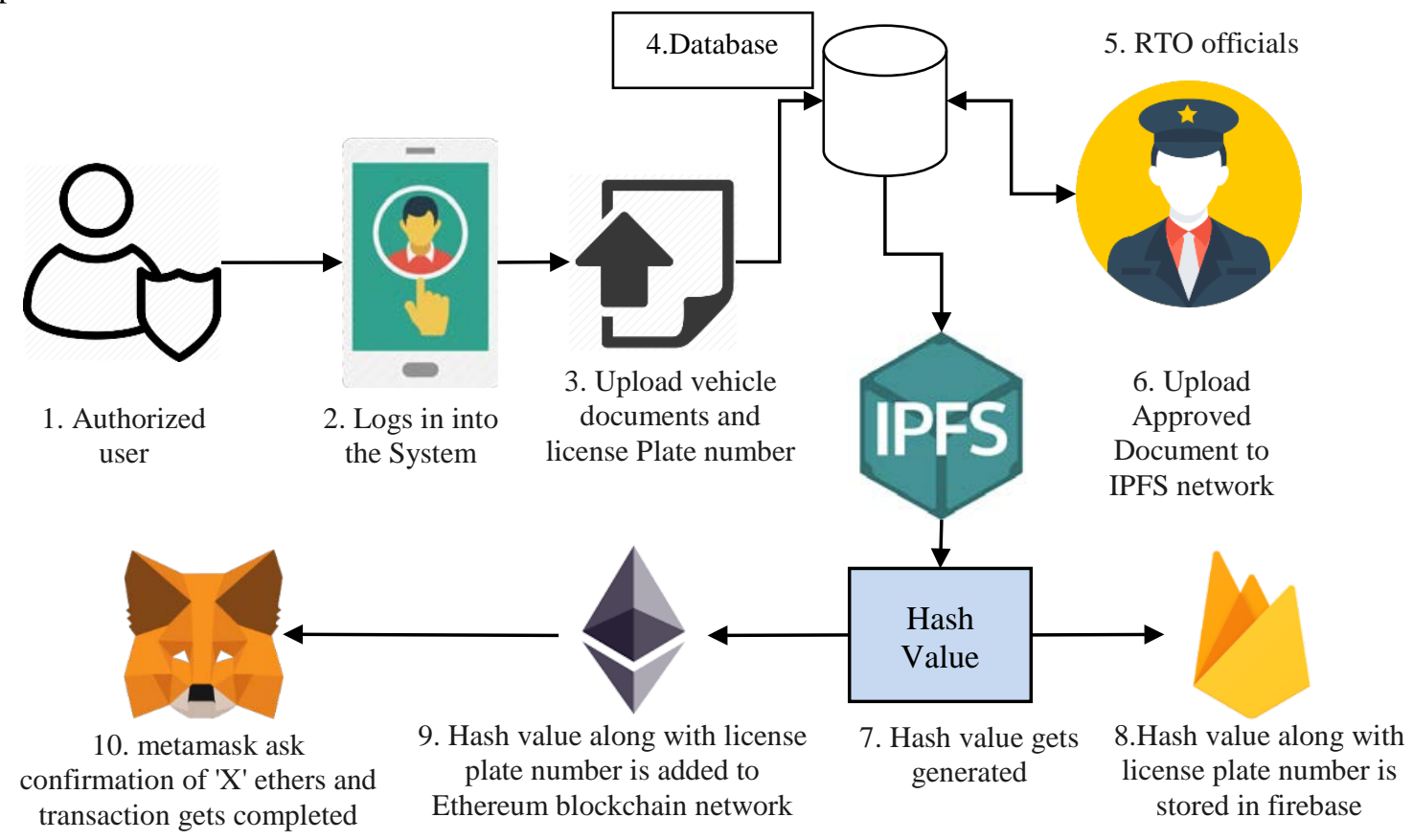

Fig. 1. Sequence of Activities for Uploading the Information

Now the user can add the required documents to the portal. The documents can be anything like RC book, driving license, insurance papers etc. For every document upload, alert is sent to the RTO officials for approval. RTO official will verify the documents and accept or reject it. On approval from RTO, final submission button gets enabled. After clicking on this button, a pop-up from MetaMask, prompts the user to confirm the transaction and then the documents are published in the InterPlanetary File System (IPFS) network. Once the user uploads documents it's converted into a buffer file (as IPFS network doesn't accept any other form). Now the buffer is sent to IPFS network and it returns IPFS hash. The generated hash is sent to Ethereum blockchain and the transaction is handled by the Metamask wallet. Simultaneously our application also adds them to the firebase database along with the user's vehicle license plate no. We assign each hash value (acquired from IPFS) with corresponding vehicle number in the database. During retrieval, we just match scanned value with each value of vehicle plate number available in the database to get appropriate user details. 
The blockchain is a distributed, decentralised system that is shared, replicated and consistent among the members of its network [14-15]. This helps to keep the data unaltered and can be managed securely [16]. Here since we were intended to develop Ethereum Application such that Ethereum transactions work could be monitored devoid of running a full Ethereum node and thus Metamask is used. Metamask gave power to work with app on browser by leveraging multiple Ethereum testnets and the Ethereum mainnet [17]. MetaMask has functionalities akin to an Ethereum wallet, it acts like a bridge between the web browser and Ethereum blockchain. All the documents are first stored in the InterPlanetary File System (IPFS) because it is prohibitively expensive to store a lot of data on the Ethereum blockchain [18-19]. The InterPlanetary File System (IPFS) is a protocol and peer-to-peer network for storing and sharing data in a distributed file system. IPFS uses content-addressing to uniquely identify each file in a global namespace connecting all computing devices. IPFS allows users to not only receive but host content, in a similar manner to BitTorrent. As opposed to a centrally located server, IPFS is built around a decentralized system of user-operators who hold a portion of the overall data, creating a resilient system of file storage and sharing. Any user in the network can serve a file by its content address, and other peers in the network can find and request that content from any node which has it, using a Distributed Hash Table (DHT).

Whenever a file is simply uploaded onto the IPFS network, it generates a unique hash value for the same. This hash value is utilized to retrieve the file from the network [20]. The key characteristics of IPFS are:

- $\quad$ Content can be distinctively identified using unique cryptographic hash identifier of the file.

- $\quad$ Files with the same content cannot be duplicated and is stored only once.

- Data is verified with its checksum, so if the hash changes, then IPFS will know that the data has been tampered.

The other platform used is a firebase, a centralized database used for integration mainly with android application. In the current implementation we are using firebase for storing the hash values associated with each of the number plates [21].

\subsection{Information access interface}

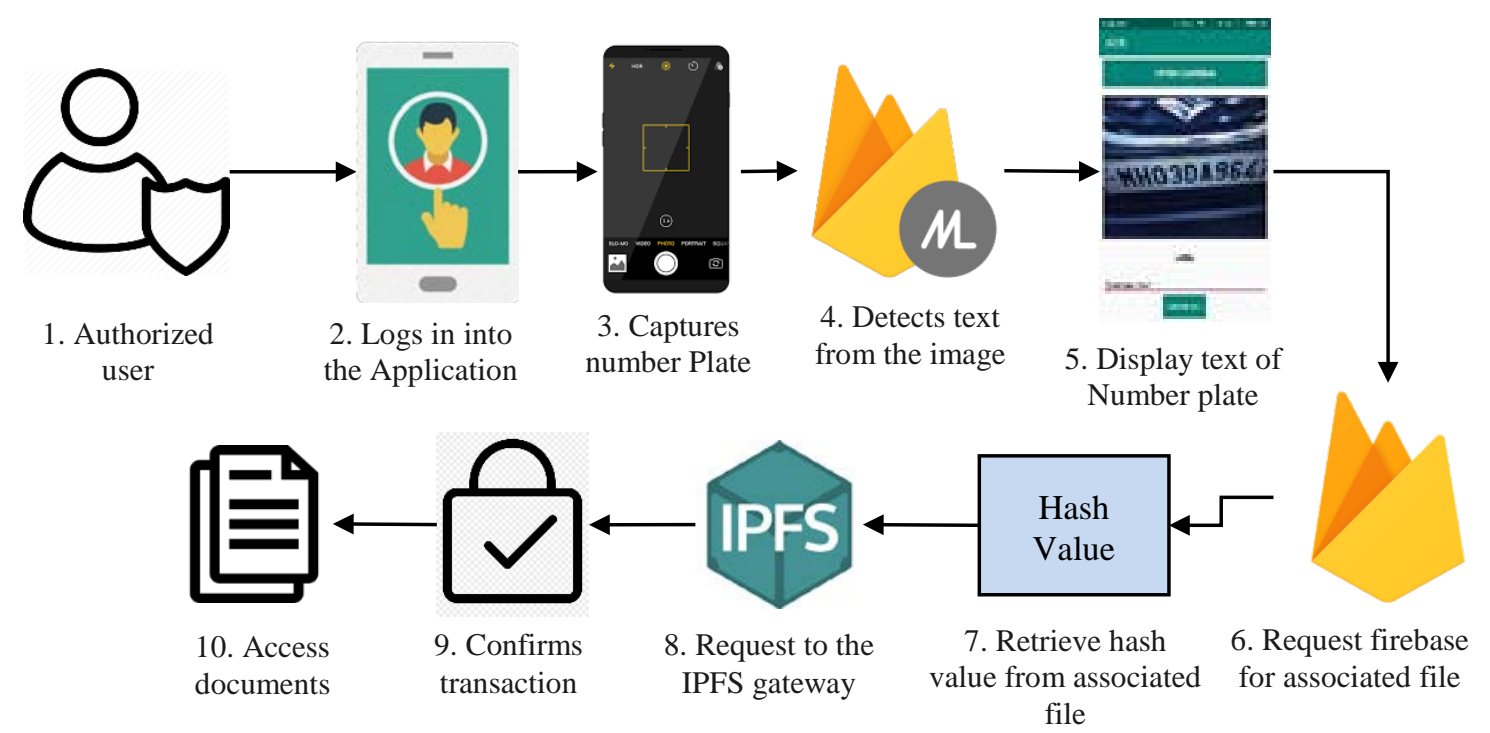

Fig. 2. Sequence of Activities for Accessing the Information

Information access interface is an android application likely to be used by traffic police officials. When traffic policemen, opens the application, he/she is prompted to login into the application. After successful login, now he/she can capture a vehicle's license plate number. After scanning, the vehicle license plate number information is retrieved as a plain text from the captured image. Firebase ML kit [22] is used for detecting text from image. The SDK comes with a collection of pre-defined capabilities that are commonly required in applications. Firebase ML kit offers machine learning capabilities underneath a form of a wrapper.

After getting the text, the text is matched with existing data in firebase database. Once the appropriate match is found, the module retrieves the required hash values and downloads the documents automatically by appending the retrieved value with the following link:

https://gateway.ipfs.io/ipfs/ 


\section{Results and Discussion}

As discussed in the introduction section, the major research objectives are

- $\quad$ R1. Develop an efficient system to identify the vehicle number

- R2. Provide easy access of all information related to particular vehicle

- R3. Maintain data security

Table. 1. Vehicle Number Plate Identified under Different Circumstances

\begin{tabular}{|c|c|c|}
\hline \multicolumn{3}{|c|}{ Light condition: Day light } \\
\hline Categories & Correctly Identified & Incorrectly Identified \\
\hline A & 29 & 1 \\
\hline B & 28 & 2 \\
\hline C & 26 & 4 \\
\hline D & 24 & 6 \\
\hline \multicolumn{3}{|c|}{ Light condition: Evening light } \\
\hline A & 29 & 1 \\
\hline B & 27 & 3 \\
\hline C & 25 & 5 \\
\hline D & 24 & 6 \\
\hline A & 27 & 6 \\
\hline B & 24 & 7 \\
\hline C & 23 & 9 \\
\hline D & 21 & 3 \\
\hline
\end{tabular}

The overall system evaluation is based on performance analysis and user experience analysis. In performance analysis, we have focused majorly on evaluating the accuracy of vehicle number recognition from the captured image of vehicle's license plate number which is our first research objective. For this purpose, we have considered the following categories:

- A: Number plate with white background and Black text

- B: Number plate with yellow background and Black text

- $\quad$ C: Number plate with white background and Black text and number written in artistic way

- D: Number plate with yellow background and Black text and number written in artistic way

To determine whether the light illumination plays any role in recognition process, we have tested all four categories (A, B, C, D) under three conditions viz.

- Day light

- $\quad$ Evening light and

- Night light

During night the license plate number image is captured is using flash light.

For each category (A,B,C,D), we have considered 30 samples. So in total, 120 samples are tested under three different light illumination conditions. Table 1 shows the number of correctly and incorrectly identified license plate numbers information under different light illumination conditions.

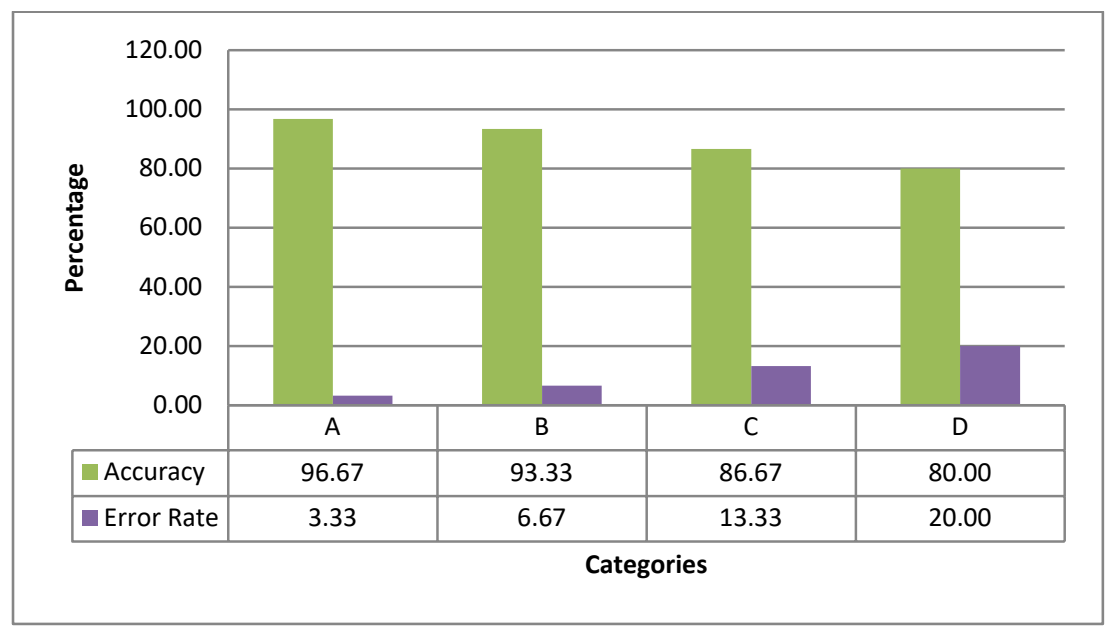

Fig. 3.Accuracy and Error Rate Graph under day light 
Figure 3, 4 and 5 shows the percentage accuracy and error rate for category A, B,C, D in day light, in evening light and in night light using flash light respectively. On comparing the three graphs shown in figure 3,4 and 5 it is observed that the accuracy of recognizing the license plate number information is highest in day light, moderate in evening light and lowest in night light where flash light of camera was used to capture the image of number plate. The error rate of number recognition is more when number plate text is written in artistic form on the license plate as compared to the normal standard text used for license plate numbers.

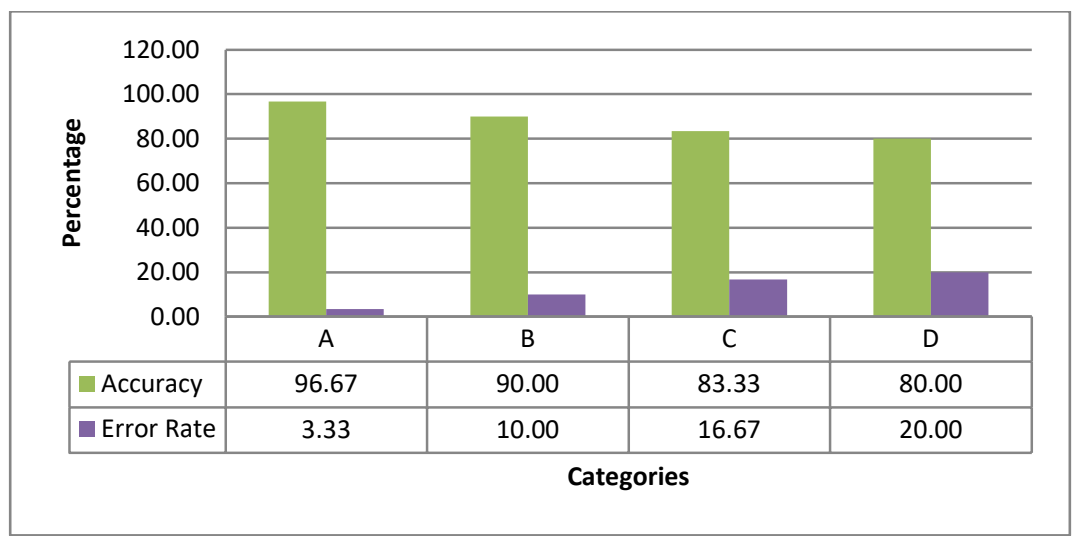

Fig. 4.Accuracy and Error Rate Graph under evening light

The average accuracy of vehicle number plate recognition during day time is $89.17 \%$ while the average error rate is $10.83 \%$. The average accuracy of vehicle number plate recognition during evening time is $87.50 \%$ while the percentage error rate is $12.50 \%$. The average accuracy of vehicle number plate recognition during night time when image is clicked in flash light is $79.17 \%$ while the average percentage error rate is $20.83 \%$. The average accuracy of overall number plate recognition module is $85.28 \%$ and average error rate is $14.72 \%$. From this we can conclude that the our research objective to design efficient number plate recognition module is achieved.

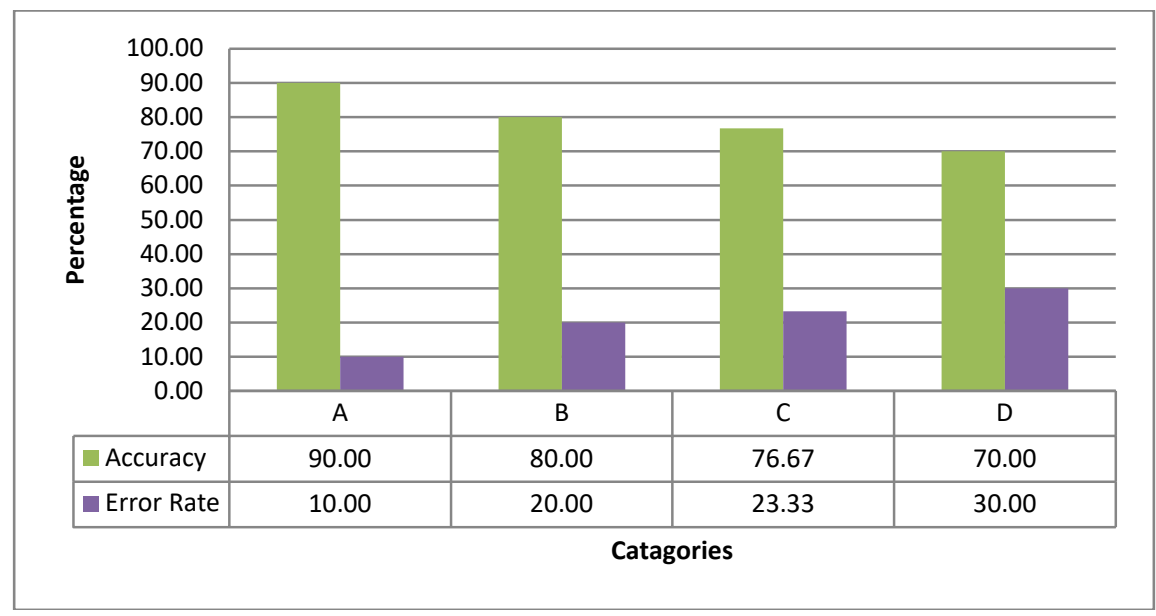

Fig. 5.Accuracy and Error Rate Graph under dark condition using flash light

Figure 6, shows few samples for number plate recognition which was captured and processed while testing the number plate recognition module. The other performance measure is to evaluate the overall user experience. When user experience is evaluated, we will come to know whether the proposed system provide easy access to the document or not which is our second research objective. To evaluate that, we have created dummy user account, uploaded dummy documents and verified that account to create a node. Later the information access module was installed on mobile handset of 10 different people and we gave them verified admin rights to access the information. These people then scanned the license plate number of the vehicle and accessed the related information. To measure the perception of users regarding usability of the system, the feedback of these 10 people is taken where they have rated the features of the system on the scale of 1-10. Table 2, gives the overview of feedback analysis. 

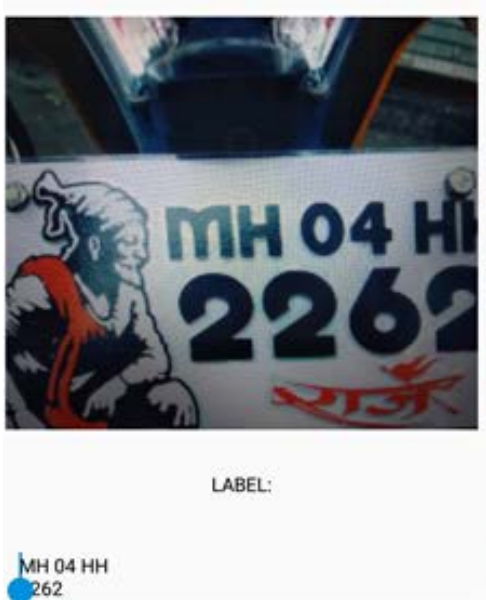

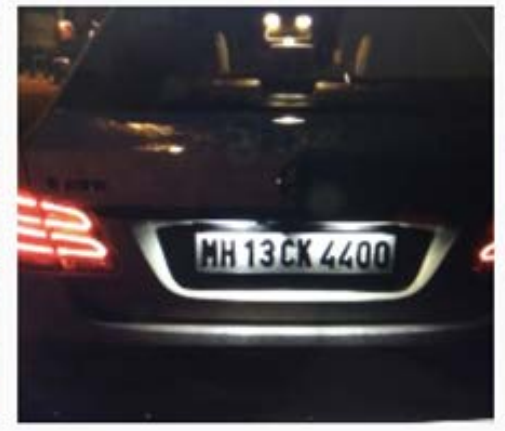

LABEL:

MH 13 CK 4400

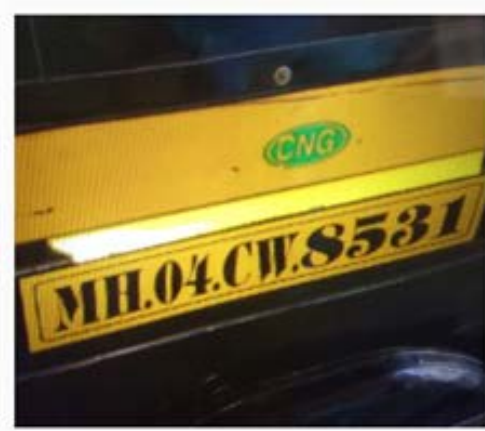

LABEL:
ENG MHO4CW.S581

Fig. 6. Samples for vehicle number plate recognition

Table 2. Feedback Analysis

\begin{tabular}{|c|l|c|}
\hline Sr. No. & Feedback Question & Average Score \\
\hline 1 & The application was easy to use & 9.37 \\
\hline 2 & No specific technical knowledge is required to use this application & 9.12 \\
\hline 3 & $\begin{array}{l}\text { Time required to recognize the number on license plate is within the acceptable time limit } \\
\text { (Acceptable time limit is maximum 20 sec) }\end{array}$ & 8.74 \\
\hline 4 & $\begin{array}{l}\text { Time required to fetch the document is within the acceptable time limit (Acceptable time } \\
\text { limit is maximum 50 sec) }\end{array}$ & 8.03 \\
\hline 5 & Rate the overall performance of the application & 8.9 \\
\hline
\end{tabular}

From the table it has been observed that users were happy with the overall experience of using the application. Thus we can say that it can be proved useful for actual police too and hence the second objective is also achieved. The third research objective is related to maintaining data security and integrity. As we have used IPFS and blockchain for maintaining information so the inherent properties of these platforms help us to achieve security. Any change in a data will result in different hash value and which won't be matching with the hash stored on blockchain. So when anyone tries to alter anything then such kind of activities can be tracked easily.

\section{Conclusion}

The Car-Docs application discussed in this paper is intended to provide the fast access of vehicle information to the authorized person (traffic police). One the traffic police side, all information about the vehicle is accessed based on vehicle number. So vehicle number plate recognition is a crucial part of this application and hence we thoroughly tested the number plate recognition module in different scenario. The average accuracy of this module is $85.28 \%$ and average error rate is $14.72 \%$. It has been also observed that accuracy of number plate recognition is more during day time than in night. The next important feature of this application is data security and developing a tamper proof system. To achieve this we have developed blockchain based architecture and use IPFC network for cost effective storage infrastructure. To evaluate the usability and user experience of the application, we have created dummy accounts with sample data and give the authorized access credential to few people and asked them to use our application. Finally feedback is taken from them which say that users were satisfied with the overall performance of the application. We believe that this application will be helpful for traffic police as well as RTO official for speedy processing of the data. Such kind of system is need of the time. It can be used to control many criminal activities. Also it can be useful to get information about unclaimed vehicles parked randomly. In future we are planning to have a module for processing the fine payment and other essential modules to make it as a complete package.

\section{References}

[1] https://www.acko.com/articles/traffic-rules-violations/how-does-the-e-challan-system-work/ Accessed on: 12 Dec 2019

[2] https://trafficpolicemumbai.maharashtra.gov.in/mumtrafficapp-2/ Accessed on: 28 Dec 2019 
[3] https://timesofindia.indiatimes.com/city/nagpur/303-traffic-cops-using-personal-phones-to-click-violators-photos/articleshow/ 56654285.cms Accessed on: 17 Jan 2020

[4] https://timesofindia.indiatimes.com/city/goa/with-body-worn-cameras-cops-to-record-interaction-with-traffic-violators/ articleshow/65677471.cms Accessed on: 17 Jan 2020

[5] https://www.business-standard.com/article/news-ani/coimbatore-traffic-police-gets-body-worn-cameras-119071800161_1.html Accessed on: 4 Feb 2020

[6] Avanish Mishra, Ashish Singh, Rajeshwar Singh, Prof. Nilima Nikam. "Smart RTO System" International Journal of Advance Research, Ideas and Innovations in Technology 3.2 (2017)

[7] Chandrakant Umarani, RashmiTeggi, Prachi Shetti, Lavanya Dodamani, Yogita Havale, " Smart RTO Web and Android Application”, International Journal of Engineering Science and Computing, Vol. 7 (6), June 2017, pp 12576-12578

[8] Alpana Gopi, Litty Rajan,Divya P R, Surya Rajan , "E-RTO Management System And Vehicle Authentication Using Rfid”, International Research Journal of Engineering and Technology (IRJET), Vol 04 (05), May 2017, pp 1218-1222

[9] Manjunath Patil, Basavaraj Madagouda, Vinod Desai, “ E-RTO Management System”, International Journal of Engineering Research \& Technology (IJERT), ISSN: 2278-0181 Vol. 2 (7), July - 2013, pp 207-2011

[10] A. Choudhury and A. Negi, "A new zone based algorithm for detection of license plate from Indian vehicle," 2016 Fourth International Conference on Parallel, Distributed and Grid Computing (PDGC), Waknaghat, 2016, pp. 370-374.

[11] A. Rabee and I. Barhumi, "License plate detection and recognition in complex scenes using mathematical morphology and support vector machines," IWSSIP 2014 Proceedings, Dubrovnik, 2014, pp. 59-62.

[12] Amruta bakale,spoorti awate,"Cross verification of vehicle and driver for RTO (IJETCSE) volume 14,Issue 2 april 2015, ISSN: 0976- 1353

[13] R. Shreyas, B. V. P. Kumar, H. B. Adithya, B. Padmaja and M. P. Sunil, "Dynamic traffic rule violation monitoring system using automatic number plate recognition with SMS feedback," 2017 2nd International Conference on Telecommunication and Networks (TEL-NET), Noida, 2017, pp. 1-5, doi: 10.1109/TEL-NET.2017.8343528.

[14] Dipti Pawade, Sagar Jape, Rahul Balasubramanian, Mihir Kulkarni, Avani Sakhapara,"Distributed Ledger Management for an Organization using Blockchains", International Journal of Education and Management Engineering(IJEME), Vol.8, No.3, pp.113, 2018.DOI: 10.5815/ijeme.2018.03.01.

[15] Pawade, Dipti \& Sakhapara, Avani \& Badgujar, Aishwarya \& Adepu, Divya \& Andrade, Melvita. (2020). Secure Online Voting System Using Biometric and Blockchain. 10.1007/978-981-32-9949-8_7.

[16] Pawade, Dipti \& Sakhapara, Avani \& Andrade, Melvita \& Badgujar, Aishwarya \& Adepu, Divya. (2019). Implementation of Fingerprint-Based Authentication System Using Blockchain: Methods and Protocols. 10.1007/978-981-13-3600-3_22.

[17] https://metamask.io/ Access on:13 Jan 2020

[18] Paik, Hye-young \& Xu, Xiwei \& Bandara, Dilum \& Lee, Sung \& Lo, Sin Kuang. (2019). Analysis of Data Management in Blockchain-based Systems: From Architecture to Governance. IEEE Access. PP. 1-1. 10.1109/ACCESS.2019.2961404.

[19] Dr. Gavin Wood "Ethereum: A Secure Decentralised Generalised Transaction Ledger Eip-150 Revision" available on:http://gavwood.com/paper.pdf

[20] https://ipfs.io/ Access on:13 Jan 2020

[21] https://firebase.google.com/ Access on:13 Jan 2020

[22] https://developers.google.com/ml-kit Access on:21 Feb 2020

\section{Authors' Profiles}

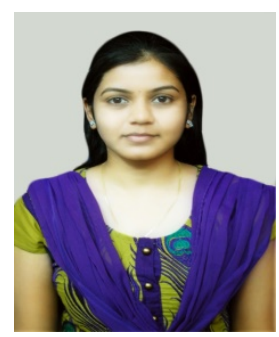

Dipti Yogesh Pawade received B.E. degree in Computer Science and Engineering from Sant Gadge Baba University in 2009 and M.E. degree in Embedded System and Computing from G. H. Raisoni College of Engineering, Nagpur in 2012. Since 2012 she is an Assistant Professor in the Department of Information Technology at K J Somaiya College of Engineering, Vidyavihar, Mumbai. Her interest includes Machine learning, Web Security, and Web Application Development.

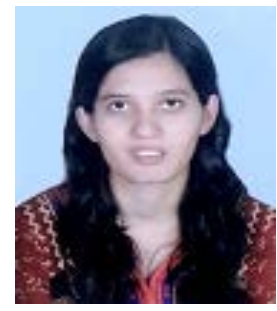

Avani Sakhapara received B.E. degree in Information Technology from Thakur College of Engineering and Technology, Mumbai University and M.E degree in Computer Engineering from K J Somaiya College of Engineering, Mumbai University. Since 2009 she is an Assistant Professor in the Department of Information Technology at K J Somaiya College of Engineering, Vidyavihar, Mumbai. Her interest includes Information Security, Machine Learning, Natural Language Processing and Web Application Development.

How to cite this paper: Dipti Pawade, Avani Sakhapara, Raj shah, Siby Thampi, Vignesh Vaid. " Blockchain Based Secure Traffic Police Assistant System ", International Journal of Education and Management Engineering (IJEME), Vol.10, No.6, pp.34-41, 2020. DOI: 10.5815/ijeme.2020.06.05 